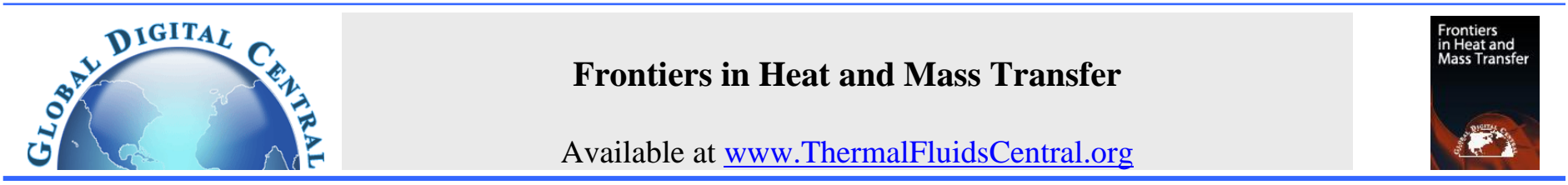

\title{
A XFEM PHASE CHANGE MODEL WITH CONVECTION
}

\author{
Dave Martin ${ }^{\mathrm{a}, \mathrm{b}, \dagger}$, Hicham Chaouki ${ }^{\mathrm{a}, \mathrm{b}}$, Jean-Loup Robert ${ }^{\mathrm{a}}$, Donald Ziegler ${ }^{\mathrm{c}}$, Mario Fafard ${ }^{\mathrm{a}, \mathrm{b}}$ \\ ${ }^{\mathrm{a}}$ Department of Civil and Water Engineering, Laval University, Quebec, QC, G1V 0A6, Canada \\ ${ }^{\mathrm{b}}$ NSERC/Alcoa Industrial Research Chair MACE 3 and Aluminium Research Centre - REGAL, Laval University, Quebec, QC, G1V 0A6, Canada \\ ${ }^{\mathrm{c}}$ Alcoa Primary Metals, Alcoa Technical Center, 100 Technical Drive, Alcoa Center, PA, 15069-0001, USA
}

\begin{abstract}
A model for two dimensional solidification problems including convection was developed by coupling the Stefan problem with the Stokes problem. The extended finite element method (XFEM) was used to capture the strong discontinuity in velocity and pressure as well as the jump in heat flux at the phase change interface. The melting temperature and no-slip condition were imposed on the interface using a Lagrange multiplier and the penalization method, respectively. The resulting formulations were then coupled using a fixed point iteration algorithm. The model was able to reproduce the benchmark simulations while maintaining a sharp phase change interface.
\end{abstract}

Keywords: Phase change, XFEM, Convection

\section{INTRODUCTION}

The finite element method Reddy (2006) has been extensively studied and successfully used in a wide variety of scenarios involving continuous media but particular situations are still problematic. The finite element method uses a polynomial interpolation within individual elements to approximate the solution. Consequently, it can only be applied to problems with discontinuities by splitting the domain into submeshes. This makes the finite element method ill suited to solve problems involving discontinuities that are part of the solution or move in time. The Stefan problem Nedjar (2002); Beckermann et al. (1999); Helenbrook (2013); Özişik (1993) for the isothermal solidification or melting of a material is one such situation because of the discontinuous heat flux at the moving phase change interface. The introduction of convection in the liquid phase adds to the discontinuous nature of the problem by requiring a zero value velocity in the solid phase and a non-zero velocity in the liquid phase.

The extended finite element method Belytschko et al. (2001); Dolbow et al. (2000); Belytschko et al. (2009) is based on the partition of unity method Babuska and Melenk (1997); Dolbow et al. (2000); Melenk and Babuska (1996). Using carefully selected functions $\psi(\mathbf{x}, t)$, the technique adds additional degrees of freedom that will "enrich" the interpolation and allows the solution to adopt a non-linear behavior. The particular type of behavior is determined by the enrichment function $\psi(\mathbf{x}, t)$, known a priori. Only nodes having support cut by the interface and have a modified behavior must be enriched (see figure 1). Consequently, the additional computational costs are local to the interface. The interface geometry is stored and transported in a computationally efficient manner, most commonly using the level set method Osher and Sethian (1988); Osher and Fedkiw (2001).

Numerous extended finite element models for the solutions of the classical (diffusive) Stefan problem are found in the literature Chessa et al. (2002); Bernauer and Herzog (2011); Merle and Dolbow (2002);

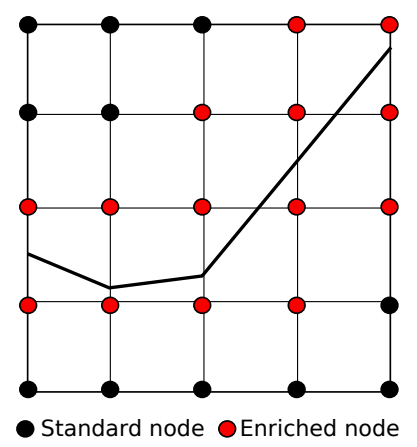

Fig. 1 Enriched node mesh to capture discontinuity $\Gamma$

Ji et al. (2002). However, most real-life scenarios involve heat and mass transfer by convection in the liquid phase Zabaras et al. (2006); Vynnycky and Kimura (2007); Brent et al. (1988). The impact of this additional contribution on the behavior of the phase change interface has been explored for dendritic solidification in Zabaras et al. (2006), using an extended finite element formulation for the Stefan problem and a phasefield formulation for the Navier-Stokes equations. To our knowledge, no coupled extended finite element formulation for both Stefan and Stokes (or Navier-Stokes) equations exists in the literature. Such an approach reduces the algorithm's complexity, as a single numerical method is used to model the discontinuities in both problems. Furthermore, the extended finite element method allows for accurate results with larger mesh sizes compared to diffused techniques.

For more complex problems, the use of different densities in the solid and liquid phases for the phase-change problem leads to a mass flux boundary in the Stokes equations. The explicit interface used in this work

${ }^{\dagger}$ Corresponding author.Email: dave.martin.1@ulaval.ca 
provides a solid framework for developing such models.

In the work presented here, a coupled formulation using the extended finite element method for both the Stefan problem and Stokes equations is developed. The convective term in the energy equation is obtained from the solution of the Stokes equations and the Boussinesq approximation is used to generate the natural convection within the liquid, where present. A fixed point iteration scheme is then used to obtain a converged solution for a given time step.

The paper is divided as follows. The governing equations for the Stefan and Stokes problems are described in section 2. The finite element formulation, level set problem and details concerning the interface movement and extended finite element method are described in section 3. Benchmark examples are then solved in section 4 to validate the algorithm. To this end, the commercial finite element simulation software Comsol was used with a moving mesh algorithm to capture the interface movement. Finally, the paper ends with some concluding remarks.

\section{GOVERNING EQUATIONS}

\subsection{Stefan Formulation}

Consider a domain $\Omega$ with an initial temperature $T\left(x, t_{0}\right)$ and interface $\Gamma$ separating solid $\left(\Omega_{s}\right)$ and liquid $\left(\Omega_{l}\right)$ phases with different thermal properties. We suppose that the density is identical in both phases and that the material has an isothermal phase change at some melting temperature $T_{m}$. Applying the conservation of energy in $\Omega$ results in equations

$$
\begin{array}{rlrl}
\left(\rho c_{p}\right)_{s} \frac{\partial T}{\partial t}-\nabla \cdot\left(k_{s} \nabla T\right) & =0 & & \mathbf{x} \in \Omega_{s} \\
\left(\rho c_{p}\right)_{l}\left(\frac{\partial T}{\partial t}+\mathbf{v} \cdot \nabla T\right)-\nabla \cdot\left(k_{l} \nabla T\right) & =0 & & \mathbf{x} \in \Omega_{l} \\
T-T_{m} & =0 & & \mathbf{x} \in \Gamma \\
T & =\hat{T} & \mathbf{x} \in \Gamma_{D} \\
-k \nabla T \cdot \mathbf{n} & =\hat{q} & & \mathbf{x} \in \Gamma_{N}
\end{array}
$$

where $c_{p}$ is the specific heat, $k$ the thermal conductivity, $\rho$ the density and $\mathbf{v}$ the liquid phase velocity. The melting temperature must be applied on the solid-liquid interface (1c). Dirichlet and Neumann type boundaries away from the interface are applied on $\partial \Omega=\Gamma_{N} \cup \Gamma_{D}$ as usual (1d,1e).

Conservation of energy at the interface requires that the jump in heat flux normal to the interface (caused by the imposition of the melting temperature) be related to the rate of solidification or melting of the material as described by

$$
\llbracket-k \nabla T \rrbracket \cdot \mathbf{n}_{\Gamma}=\left(k_{l} \nabla T_{l}-k_{s} \nabla T_{s}\right) \cdot \mathbf{n}_{\Gamma}=\rho L v_{\Gamma} \quad \mathbf{x} \in \Gamma
$$

where $L$ is the latent heat and $v_{\Gamma}$ the normal interface velocity Özişik (1993). The normal vector $\mathbf{n}_{\Gamma}$ points from the liquid to solid phase, meaning that the interface velocity is positive for melting and negative for solidification.

Tracking the moving interface is done using the level set method Osher and Fedkiw (2001); Osher and Sethian (1988). The principle behind this method is to introduce a new variable $\phi(\mathbf{x}, t)$ defined as the signed distance function to the interface:

$$
\phi(\mathbf{x}, t)=\min _{\mathbf{x}_{\Gamma} \in \Gamma}\left|\mathbf{x}-\mathbf{x}_{\Gamma}(t)\right| \operatorname{sign}\left(\mathbf{n}_{\Gamma} \cdot\left(\mathbf{x}-\mathbf{x}_{\Gamma}(t)\right)\right) \quad \mathbf{x} \in \Omega
$$

The interface is then easily identified as the set of points where $\phi(\mathbf{x}, t)=$ 0 . In this work, the level set field is constructed so that the liquid phase is on the positive side of the interface (i.e. $\mathbf{x} \in \Omega_{l}$ if $\phi(\mathbf{x}, t)>0$ ).

\subsection{Stokes formulation}

In the present study, the liquid phase velocity $\mathbf{v}$ is governed by the Stokes problem for viscous incompressible fluids:

$$
\begin{array}{rlrl}
\rho \frac{\partial \mathbf{v}}{\partial t}-\nabla \cdot \boldsymbol{\sigma}-\mathbf{f}_{b} & =0 & & \mathbf{x} \in \Omega_{l} \\
\nabla \cdot \mathbf{v} & =0 & \mathbf{x} \in \Omega_{l} \\
\mathbf{v} & =0 & \mathbf{x} \in \Gamma \\
\mathbf{v} & =\hat{\mathbf{v}} & \mathbf{x} \in \Gamma_{D} \\
\boldsymbol{\sigma} \cdot \mathbf{n} & =\hat{\boldsymbol{\sigma}} & \mathbf{x} \in \Gamma_{N} \\
\mathbf{f}_{b} & =\rho \beta\left(T-T_{m}\right) \mathbf{g} & & \\
\boldsymbol{\sigma} & =-p \mathbf{I}+2 \mu D(\mathbf{v}) & \\
D(\mathbf{v}) & =\frac{1}{2}\left(\nabla \mathbf{v}+\nabla \mathbf{v}^{T}\right) & &
\end{array}
$$

where $p$ is the pressure, $\mu$ the viscosity, $\mathbf{f}_{b}$ the buoyancy source term, $\beta$ the thermal expansion coefficient and $D(\mathbf{v})$ the rate of deformation tensor.

The buoyancy force $\mathbf{f}_{b}$ term will create natural convection currents caused by variations in temperature. The density is assumed constant and identical for both phases (Boussinesq approximation) so no mass flux is present at the interface and a no-slip condition is applied (4c). The other physical properties are assumed constant. The initial velocity field $\mathbf{v}\left(\mathbf{x}, t_{0}\right)$ is assumed divergence-free with a given initial pressure field $p\left(\mathbf{x}, t_{0}\right)$. Dirichlet and Neumann type boundaries away from the interface are applied on $\partial \Omega=\Gamma_{N} \cup \Gamma_{D}$ as usual $(4 \mathrm{~d}, 4 \mathrm{e})$.

The convection term in the complete Navier-Stokes equations was removed, leading to two linear systems of equations for the heat transfer and fluid flow problems. The only non-linearity is in the coupling terms between the two problems; the convective heat transfer and buoyancy force.

\subsection{Enriched Interpolation Scheme}

To account for the jump in heat flux at the interface, the temperature gradient must be discontinuous. Furthermore, the application of the interface boundary condition (1c), implies that the temperature is continuous at the interface. This behavior is captured by using the approximation Chessa et al. (2002)

$$
\begin{aligned}
T(\mathbf{x}, t) & =\sum_{i \in I} N_{i}^{T}(\mathbf{x}) T_{i}(t)+\sum_{j \in J} N_{j}^{T}(\mathbf{x}) \psi_{j}^{T}(\mathbf{x}, t) T_{j}^{*}(t) \\
\psi_{j}^{T}(\mathbf{x}, t) & =|\phi(\mathbf{x}, t)|-\left|\phi\left(\mathbf{x}_{j}, t\right)\right|
\end{aligned}
$$

where $N^{T}$ are the standard interpolation functions, $T_{i}$ and $T_{j}^{*}$ the standard and enriched degrees of freedom, respectively, and $\psi_{j}^{T}(\mathbf{x}, t)$ the enrichment function, based on the absolute value of the level set field. A more compact way to write (5) is by use the more standard matrix form

$$
\begin{aligned}
T(\mathbf{x}, t) & =\left\langle N_{T}\right\rangle\{T\} \\
\left\langle N_{T}\right\rangle & =\left\langle N_{1}^{T}, N_{2}^{T}, \ldots, N_{1}^{T} \psi_{1}^{T}, N_{2}^{T} \psi_{2}^{T}, \ldots\right\rangle \\
\{T\} & =\left\langle T_{1}, T_{2}, \ldots, T_{1}^{*}, T_{2}^{*}, \ldots\right\rangle^{T}
\end{aligned}
$$

When using (5) special attention must be given to elements containing enriched nodes that are not cut by the interface, called blending elements. A modified interpolation scheme must be used in these elements to maintain an optimal convergence rate, as described in Fries (2008); Shibanuma and Utsunomiya (2009).

In order to capture the jump in the heat flux at the interface, a Lagrange multiplier $\mathbf{q}$ will be used Gerstenberger (2010). The interpolation 
scheme for the Lagrange multiplier is given by

$$
\begin{aligned}
\mathbf{q}(\mathbf{x}, t) & =\sum_{i \in I} N_{i}^{q}(\mathbf{x}) \mathbf{q}_{i}(t)+\sum_{j \in J} N_{j}^{q}(\mathbf{x}) \psi_{j}^{q}(\mathbf{x}, t) \mathbf{q}_{j}^{*}(t) \\
\psi_{j}^{q}(\mathbf{x}, t) & =H(\phi(\mathbf{x}, t))-H\left(\phi\left(\mathbf{x}_{j}, t\right)\right) \\
H(\mathbf{x}, t) & = \begin{cases}1 & \text { if } \phi(\mathbf{x}, t)<0 \\
0 & \text { if } \phi(\mathbf{x}, t)>0\end{cases}
\end{aligned}
$$

where $\mathrm{H}$ is a modified Heaviside function.

Following (6), the Lagrange multiplier may be rewritten as

$$
\begin{aligned}
\mathbf{q}(\mathbf{x}, t) & =\left[N_{q}\right]\{\mathbf{q}\} \\
{\left[N_{q}\right] } & =\left[\begin{array}{cccccc}
N_{1}^{q} & \ldots & N_{d}^{q} \psi_{d}^{q} & 0 & \ldots & 0 \\
0 & \ldots & 0 & N_{1}^{q} & \ldots & N_{d}^{q} \psi_{d}^{q}
\end{array}\right] \\
\{\mathbf{q}\} & =\left\langle q_{1}^{x}, \ldots, q_{d}^{x *}, q_{1}^{y}, \ldots, q_{d}^{y *}\right\rangle^{T}
\end{aligned}
$$

where $\left[N_{q}\right]$ is the matrix of interpolation functions.

The Stokes equations are valid (and solved) in the liquid phase only. For this purpose, the fluid-structure interaction approach, proposed in Gerstenberger and Wall (2008), is used. The velocity and pressure fields are interpolated using the following scheme:

$$
\begin{aligned}
\mathbf{v}(\mathbf{x}, t) & =\sum_{i \in I} N_{i}^{v}(\mathbf{x}) \psi^{v}(\mathbf{x}, t) \mathbf{v}_{i}(t) \\
p(\mathbf{x}, t) & =\sum_{i \in I} N_{i}^{p}(\mathbf{x}) \psi^{v}(\mathbf{x}, t) p_{i}(t) \\
\psi^{v}(\mathbf{x}, t) & = \begin{cases}1 & \text { if } \phi(\mathbf{x}, t)>0 \\
0 & \text { if } \phi(\mathbf{x}, t)<0\end{cases}
\end{aligned}
$$

Following (6) and (8), the velocity and pressure fields may be rewritten as:

$$
\begin{aligned}
& \mathbf{v}(\mathbf{x}, t)=\left[N_{v}\right]\{\mathbf{v}\} \\
& p(\mathbf{x}, t)=\left\langle N_{p}\right\rangle\{p\} \\
& {\left[N_{v}\right]=\left[\begin{array}{cccccc}
N_{1}^{v} & \ldots & N_{d}^{v} \psi_{d}^{v} & 0 & \ldots & 0 \\
0 & \ldots & 0 & N_{1}^{v} & \ldots & N_{d}^{v} \psi_{d}^{v}
\end{array}\right]} \\
& \{\mathbf{v}\}=\left\langle v_{1}^{x}, \ldots, v_{d}^{x *}, v_{1}^{y}, \ldots, v_{d}^{y *}\right\rangle^{T} \\
& \left\langle N_{p}\right\rangle=\left\langle N_{1}^{p}, N_{2}^{p}, \ldots, N_{1}^{p} \psi_{1}^{v}, N_{2}^{p} \psi_{2}^{v}, \ldots\right\rangle \\
& \{p\}=\left\langle p_{1}, p_{2}, \ldots, p_{1}^{*}, p_{2}^{*}, \ldots\right\rangle^{T}
\end{aligned}
$$

When using this interpolation scheme, the solid part of the domain is ignored. Also, enriched degrees of freedom are not required because no new information (behavior) is introduced. All velocity and pressure degrees of freedom whose support is completely inside the solid domain are removed from the system of equations.

\section{NUMERICAL IMPLEMENTATION}

\subsection{Stefan Problem}

The weak form of the energy conservation equations $(1 \mathrm{a}, 1 \mathrm{~b})$ is

$$
\int_{\Omega} \delta T \rho c_{p} \frac{\partial T}{\partial t} \mathrm{~d} \Omega+\int_{\Omega_{l}} \delta T \rho c_{p} \mathbf{v} \cdot \nabla T \mathrm{~d} \Omega+\int_{\Omega} \nabla \delta T k \nabla T \mathrm{~d} \Omega=0
$$

where $\delta T$ is the temperature test function. The Neumann boundary condition has been omitted for the sake of clarity. To apply the melting temperature $T_{m}(1 \mathrm{c})$, a stable Lagrange multiplier formulation is used, originally developed in Gerstenberger and Wall (2010); Baiges et al. (2012) and applied to the Stefan problem in Martin et al. (2016). The weak form of the resulting equations is

$$
\begin{gathered}
\int_{\Omega} \delta T \rho c_{p} \frac{\partial T}{\partial t} \mathrm{~d} \Omega+\int_{\Omega_{l}} \delta T \rho c_{p} \mathbf{v} \cdot \nabla T \mathrm{~d} \Omega+\int_{\Omega} \nabla \delta T k \nabla T \mathrm{~d} \Omega \\
-\int_{\Gamma} \delta T \mathbf{q} \cdot \mathbf{n}_{\Gamma} \mathrm{d} \Gamma=0 \\
\int_{\Omega} \delta \mathbf{q} \cdot\left(\frac{1}{k} \mathbf{q}+\nabla T\right) \mathrm{d} \Omega-\int_{\Gamma} \delta \mathbf{q} \cdot \mathbf{n}_{\Gamma}\left(T-T_{m}\right) \mathrm{d} \Gamma=0
\end{gathered}
$$

where $\delta \mathbf{q}$ is the test functions for the Lagrange multiplier. The Lagrange multiplier $\mathbf{q}$ is defined as a vectorial flux and interpolated on the same mesh as the temperature field. The projection of this secondary variable on the interface is then used as a scalar Lagrange multiplier to impose the melting temperature. This formulation has the advantage of being stable without the use of additional stabilization terms Gerstenberger and Wall (2010) and allows an efficient and precise evaluation of the interface flux jump Martin et al. (2016), required to evaluate the interface velocity (2).

Using a backward Euler scheme for the time derivative of $\mathrm{T}$ Fries and Zilian (2009) in (12) gives:

$\int_{\Omega} \delta T^{n+1} \rho \frac{\left(c_{p} T\right)^{n+1}-\left(c_{p} T\right)^{n}}{\Delta t} \mathrm{~d} \Omega+\int_{\Omega_{l}} \delta T^{n+1} \rho\left(c_{p} \mathbf{v} \cdot \nabla T\right)^{n+1} \mathrm{~d} \Omega$

$+\int_{\Omega} \nabla \delta T^{n+1}(k \nabla T)^{n+1} \mathrm{~d} \Omega-\int_{\Gamma} \delta T^{n+1} \mathbf{q}^{n+1} \cdot \mathbf{n}_{\Gamma} \mathrm{d} \Gamma=0$

$\int_{\Omega} \delta \mathbf{q}^{n+1} \cdot\left(\frac{1}{k} \mathbf{q}+\nabla T\right)^{n+1} \mathrm{~d} \Omega$

$-\int_{\Gamma} \delta \mathbf{q}^{n+1} \cdot \mathbf{n}_{\Gamma}\left(T^{n+1}-T_{m}\right) \mathrm{d} \Gamma=0$

where $n$ indicates the previous time step. Note that because the enriched interpolation functions vary in time, the test functions $\delta T$ and $\delta \mathbf{q}$ will vary in time as well and must be evaluated at the current time step in (13).

After replacing $T$ and $\mathbf{q}$ with their approximations we obtain the system of equations

$$
\begin{aligned}
& +\left\{\begin{array}{c}
0 \\
\left\{\mathbf{f}_{l}\right\}
\end{array}\right\}=0 \\
& {[M]=\frac{1}{\Delta t} \sum_{e} \int_{\Omega^{e}}\left\{N_{T}\right\}^{n+1} \rho c_{p}^{n+1}\left\langle N_{T}\right\rangle^{n+1} \mathrm{~d} \Omega} \\
& {[M]^{*}=\frac{1}{\Delta t} \sum_{e} \int_{\Omega^{e}}\left\{N_{T}\right\}^{n+1} \rho c_{p}^{n}\left\langle N_{T}\right\rangle^{n} \mathrm{~d} \Omega} \\
& {[C]=\sum_{e} \int_{\Omega_{l}^{e}}\left\{N_{T}\right\}^{n+1} \rho c_{p}^{n+1} \mathbf{v}^{n+1}\left[B_{T}\right]^{n+1} \mathrm{~d} \Omega} \\
& {[K]=\sum_{e} \int_{\Omega^{e}}\left(\left[B_{T}\right]^{T}\right)^{n+1} k^{n+1}\left[B_{T}\right]^{n+1} \mathrm{~d} \Omega} \\
& {\left[M_{q}\right]=\sum_{e} \int_{\Omega^{e}}\left(\left[N_{q}\right]^{T}\right)^{n+1} \frac{1}{k^{n+1}}\left[N_{q}\right]^{n+1} \mathrm{~d} \Omega} \\
& {[Q]=\sum_{e} \int_{\Omega^{e}}\left(\left[N_{q}\right]^{T}\right)^{n+1}\left[B_{T}\right]^{n+1} \mathrm{~d} \Omega} \\
& {[L]=\sum_{e} \int_{\Gamma^{e}}\left\{N_{T}\right\}^{n+1}\left[N_{q}\right]^{n+1} \mathbf{n}_{\Gamma} \mathrm{d} \Gamma} \\
& \left\{\mathbf{f}_{l}\right\}=\sum_{e} \int_{\Gamma^{e}}\left(\left[N_{q}\right]^{T}\right)^{n+1} \mathbf{n}_{\Gamma} T_{m} \mathrm{~d} \Gamma
\end{aligned}
$$$$
\left[\begin{array}{cc}
{[M]+[C]+[K]} & -[L] \\
{[Q]-[L]^{T}} & {\left[M_{q}\right]}
\end{array}\right]\left\{\begin{array}{l}
\{T\}^{n+1} \\
\{\mathbf{q}\}^{n+1}
\end{array}\right\}-\left[\begin{array}{cc}
{[M]^{*}} & 0 \\
0 & 0
\end{array}\right]\left\{\begin{array}{l}
\{T\}^{n} \\
\{\mathbf{q}\}^{n}
\end{array}\right\}
$$ 
where $B_{i j}=\frac{\partial N_{j}}{\partial x_{i}}$ is the gradient matrix. In elements which are not cut by the interface, the Lagrange multiplier is weakly coupled with the temperature gradient but no constraint is present and the system reduces to:

$$
\left[\begin{array}{cc}
{[M]+[C]+[K]} & 0 \\
{[Q]} & {\left[M_{q}\right]}
\end{array}\right]\left\{\begin{array}{l}
\{T\}^{n+1} \\
\{\mathbf{q}\}^{n+1}
\end{array}\right\}-\left[\begin{array}{cc}
{[M]^{*}} & 0 \\
0 & 0
\end{array}\right]\left\{\begin{array}{l}
\{T\}^{n} \\
\{\mathbf{q}\}^{n}
\end{array}\right\}=0
$$

\subsection{Stokes Problem}

The weak form of the Stokes problem (4) is given as follows

$$
\begin{aligned}
& \int_{\Omega_{l}} \delta \mathbf{v} \cdot \rho \frac{\partial \mathbf{v}}{\partial t} \mathrm{~d} \Omega+\int_{\Omega_{l}} 2 \mu D(\delta \mathbf{v}): D(\mathbf{v}) \mathrm{d} \Omega-\int_{\Omega_{l}}(\nabla \cdot \delta \mathbf{v}) p \mathrm{~d} \Omega \\
& +\int_{\Omega_{l}} \delta \mathbf{v} \cdot \mathbf{f}_{b} \mathrm{~d} \Omega=0 \\
& \int_{\Omega_{l}} \delta p \nabla \cdot \mathbf{v} \mathrm{d} \Omega=0
\end{aligned}
$$

where $\delta \mathbf{v}$ and $\delta p$ are the test functions for the velocity and pressure, respectively. The Neumann boundary condition has been omitted for the sake of clarity. Using a backward Euler scheme for the time derivative of $\mathbf{v}$ Fries and Zilian (2009) in (16) gives the system of equations:

$$
\begin{aligned}
& \int_{\Omega_{l}} \delta \mathbf{v}^{n+1} \cdot \rho \frac{1}{\Delta t}\left(\mathbf{v}^{n+1}-\mathbf{v}^{n}\right) \mathrm{d} \Omega+\int_{\Omega_{l}} 2 \mu D\left(\delta \mathbf{v}^{n+1}\right): D\left(\mathbf{v}^{n+1}\right) \mathrm{d} \Omega \\
& -\int_{\Omega_{l}} \nabla \cdot \delta \mathbf{v}^{n+1} p^{n+1} \mathrm{~d} \Omega+\int_{\Omega_{l}} \delta \mathbf{v}^{n+1} \cdot \mathbf{f}_{b}^{n+1} \mathrm{~d} \Omega=0 \\
& \int_{\Omega_{l}} \delta p^{n+1} \nabla \cdot \mathbf{v}^{n+1} \mathrm{~d} \Omega=0
\end{aligned}
$$

Substituting the approximation for the velocity and pressure fields into (17) leads to the finite element system of equations

$$
\begin{aligned}
& {\left[\begin{array}{cc}
{[K]} & -[D] \\
{[D]^{T}} & 0
\end{array}\right]\left\{\begin{array}{l}
\{\mathbf{v}\}^{n+1} \\
\{p\}^{n+1}
\end{array}\right\}-\left[\begin{array}{cc}
{[M]^{*}} & 0 \\
0 & 0
\end{array}\right]\left\{\begin{array}{l}
\{\mathbf{v}\}^{n} \\
\{p\}^{n}
\end{array}\right\}+\left\{\begin{array}{c}
\left\{\mathbf{f}_{b}\right\} \\
0
\end{array}\right\}=0} \\
& {[K]=[M]+\left[\begin{array}{cc}
{\left[A_{11}\right]} & {\left[A_{12}\right]} \\
{\left[A_{12}\right]^{T}} & {\left[A_{22}\right]}
\end{array}\right]} \\
& {[M]=\frac{1}{\Delta t} \sum_{e} \int_{\Omega^{e}}\left(\left[N_{v}\right]^{T}\right)^{n+1} \rho\left[N_{v}\right]^{n+1} \mathrm{~d} \Omega} \\
& {[M]^{*}=\frac{1}{\Delta t} \sum_{e} \int_{\Omega^{e}}\left(\left[N_{v}\right]^{T}\right)^{n+1} \rho\left[N_{v}\right]^{n} \mathrm{~d} \Omega} \\
& {\left[A_{11}\right]=\sum_{e} \int_{\Omega^{e}} 2 \mu\left(\left\{B_{x}\right\}^{n+1}\left\langle B_{x}\right\rangle^{n+1}+\frac{1}{2}\left\{B_{y}\right\}^{n+1}\left\langle B_{y}\right\rangle^{n+1}\right) \mathrm{d} \Omega} \\
& {\left[A_{22}\right]=\sum_{e} \int_{\Omega^{e}} 2 \mu\left(\frac{1}{2}\left\{B_{x}\right\}^{n+1}\left\langle B_{x}\right\rangle^{n+1}+\left\{B_{y}\right\}^{n+1}\left\langle B_{y}\right\rangle^{n+1}\right) \mathrm{d} \Omega} \\
& {\left[A_{12}\right]=\sum_{e} \int_{\Omega^{e}} 2 \mu\left(\frac{1}{2}\left\{B_{y}\right\}^{n+1}\left\langle B_{x}\right\rangle^{n+1}\right) \mathrm{d} \Omega} \\
& {[D]=\sum_{e} \int_{\Omega^{e}}\left\langle\left\langle B_{x}\right\rangle^{n+1}\left\langle B_{y}\right\rangle^{n+1}\right\rangle^{T}\left\langle N_{p}\right\rangle^{n+1} \mathrm{~d} \Omega} \\
& \left\{\mathbf{f}_{b}\right\}=\sum_{e} \int_{\Omega_{l}^{e}}\left[N_{v}\right]^{n+1} \rho \beta\left(T^{n+1}-T_{m}\right) \mathbf{g} \mathrm{d} \Omega
\end{aligned}
$$

The no-slip interface boundary condition is imposed using the penalty method Chessa et al. (2002); Bernauer and Herzog (2011). This technique multiplies the residual form of equation $(4 \mathrm{c})$ by a very large penalization parameter $\lambda$ and introduces it in the finite element formulation of the momentum equation. This method is simple to implement and has proven to be robust for a variety of problems. The formulation for elements intersected by the interface becomes:

$$
\left[\begin{array}{cc}
{\left[K^{\prime}\right]} & -[D] \\
{[D]^{T}} & 0
\end{array}\right]\left\{\begin{array}{l}
\{\mathbf{v}\}^{n+1} \\
\{p\}^{n+1}
\end{array}\right\}-\left[\begin{array}{cc}
{[M]^{*}} & 0 \\
0 & 0
\end{array}\right]\left\{\begin{array}{l}
\{\mathbf{v}\}^{n} \\
\{p\}^{n}
\end{array}\right\}+\left\{\begin{array}{c}
\left\{\mathbf{f}_{b}\right\} \\
0
\end{array}\right\}=0
$$

$$
\begin{aligned}
& {\left[K^{\prime}\right]=[K]+[P]} \\
& {[P]=\sum_{e} \int_{\Gamma^{e}}\left(\left[N_{v}\right]^{T}\right)^{n+1} \lambda\left[N_{v}\right]^{n+1} \mathrm{~d} \Gamma}
\end{aligned}
$$

To solve (18) and (19) the interpolation functions for the velocity and pressure fields must satisfy the (inf-sup) condition. In this work, a pair of stable $\mathrm{Q}_{2}-\mathrm{Q}_{1}$ quadrilateral elements was used for the velocity and pressure fields, respectively.

The interpolation scheme (9) is known to cause problems when the physical domain (liquid phase) covers a very small area of the node's support Lang et al. (2014). The small contribution of the concerned degree of freedom causes a significant increase in the condition number of the global system Lang et al. (2014), leading to divergent solutions. An efficient solution was developed in Lang et al. (2014). When a degree of freedom's contribution to the system is too small, it is removed from the system. The criteria for removing a degree of freedom is Lang et al. (2014)

$$
\left(\max _{e \in E_{i}} \frac{\int_{\Omega_{l}^{e}} N_{i}(\mathbf{x}) \mathrm{d} \Omega}{\int_{\Omega^{e}} N_{i}(\mathbf{x}) \mathrm{d} \Omega}\right)^{-\frac{1}{2}}>T_{t o l}
$$

where $E_{i}$ is the set of elements connected to node i, $\Omega_{l}^{e}$ the liquid domain area in the element, $\Omega^{e}$ the element area, $N_{i}(\mathbf{x})$ the interpolation function and $T_{t o l}$ a user defined tolerance value. The greater the value for $T_{t o l}$, the smaller the contribution of the degree of freedom can be before it is removed.

The stopping criteria (20) is used on a stabilized $\mathrm{Q}_{1}-\mathrm{Q}_{1}$ in Lang et al. (2014), meaning that the velocity and pressure interpolation functions are identical, bi-linear and positive-semidefinite. The quadratic interpolation used for velocity in this work however, is not positive-semidefinite. This means that certain interface positions would lead to near zero integrals in (20) even when the liquid area is large, because the negative-valued areas of the interpolation would cancel out the positive-valued areas. To maintain the original objective of evaluating the relative contribution of the degree of freedom to the complete element, a modified criteria was used, given by equation

$$
\left(\max _{e \in E_{i}} \frac{\int_{\Omega_{l}^{e}}\left|N_{i}(\mathbf{x})\right| \mathrm{d} \Omega}{\int_{\Omega^{e}}\left|N_{i}(\mathbf{x})\right| \mathrm{d} \Omega}\right)^{-\frac{1}{2}}>T_{t o l}
$$

where the absolute value of the interpolation function is used.

Another impact of the use of a $\mathrm{Q}_{2}-\mathrm{Q}_{1}$ interpolation scheme is the difference in integral values for the velocity and pressure fields because of the different interpolation orders. Consequently, an identical liquid phase area will lead to different values in (20) or (21) and certain nodes may have only one variable (velocity or pressure) removed. To alleviate this problem, different values of $T_{t o l}$ were used for the velocity and pressure variables and were chosen to increase the probability that if one variable is removed, so is the other.

Furthermore, in Lang et al. (2014) a preconditioner is applied to the global system before solving, allowing the use of a higher value of $T_{t o l}$ while maintaining an optimal condition number and accurate solution. Considering the relatively heuristic modifications made to the removal of degrees of freedom caused by the use of a $\mathrm{Q}_{2}-\mathrm{Q}_{1}$ formulation and to simplify the implementation of our model, the preconditioner was not applied in this work. 
The systems of equations (14) and (18) are coupled through the convection and buoyancy terms, respectively. To obtain a converged solution for both systems, a fixed point iteration scheme is used. The basic approach is to alternate between the two problems, using the updated solution of each problem when solving the other. Once the residuals of both problems, using the most recent solution, are below a certain stopping criteria, the global problem is considered converged and the algorithm proceeds to the next time step.

\subsection{Level Set Formulation}

Once an initial value $\phi\left(\mathbf{x}, t_{0}\right)$ is defined, the interface movement is governed by its transport equation

$$
\begin{gathered}
\frac{\partial \phi}{\partial t}+\mathbf{v} \cdot \nabla \phi=\frac{\partial \phi}{\partial t}+F\|\nabla \phi\|=0 \\
F=\frac{\nabla \phi}{\|\nabla \phi\|} \cdot \mathbf{v}
\end{gathered}
$$

where $\mathbf{v}$ is the convection velocity and $\mathrm{F}$ is the interface speed in the normal direction. The calculation of $\mathrm{F}$ is explained below.

Equation (22) is solved explicitly (forward Euler scheme) with the finite element method using a linear interpolation. The weak formulation and time discretization of (22) is given as follows:

$$
\int_{\Omega} \delta \phi \frac{\phi^{n+1}-\phi^{n}}{\Delta t} \mathrm{~d} \Omega+\int_{\Omega} \delta \phi F^{n}\left\|\nabla \phi^{n}\right\| \mathrm{d} \Omega=0
$$

In most applications, the normal component $\mathrm{F}$ is only known on $\Gamma$. In order to solve (23) on $\Omega$, a valid value for $F$ must first be constructed on the entire domain using Chessa et al. (2002):

$$
\begin{array}{ll}
\operatorname{sign}(\phi) \nabla F \cdot \nabla \phi=0 & \mathbf{x} \in \Omega \\
F(\mathbf{x}, t)=\frac{\nabla \phi}{\|\nabla \phi\|} \cdot \mathbf{v}_{\Gamma} & \mathbf{x} \in \Gamma
\end{array}
$$

This approach guarantees that the $\phi$ field velocity is everywhere normal to the interface and is coherent with the interface's physically determined velocity. For more details concerning the construction of $\mathrm{F}$ see Osher and Fedkiw (2003); Chessa et al. (2002). In this paper, the interface velocity is based on the jump in heat flux across the phase change boundary and is described in the following section.

Equation (23) is first order hyperbolic and must be stabilized to minimize the presence of oscillations in the solution Chessa et al. (2002); Bernauer and Herzog (2011). The GLS method is used here Hughes et al. (1989). The level set method offers several advantages. It is easily extensible to three dimensions and stores the interface location as a scalar variable. Furthermore, the level set field can be defined in a small region surrounding the interface and the level set formulation solved locally, reducing the impact on the total simulation computation time. It is also robust enough to handle interface merging and breaking naturally Osher and Fedkiw (2001).

The main disadvantage of the the level set method is its tendency to deviate from a signed distance function over time Osher and Fedkiw (2001). This error accumulates with additional time steps and degrades the quality of the solution, particularly the level set gradient near the interface. This distortion can be a source of error in the numerical solution of the level set formulation and the physical problem on which it is based. Therefore, it is necessary to reinitialize $\phi(\mathbf{x}, t)$ regularly to maintain an acceptable solution $(\|\nabla \phi\| \approx 1)$. Another limitation to the algorithm presented here is the use of an explicit time scheme for the level set formulation, which limits the size of the time step. The explicit time step is required in order to determine the nodes to enrich. In other words, the interface position must be determined before systems (14) and (18) are solved.

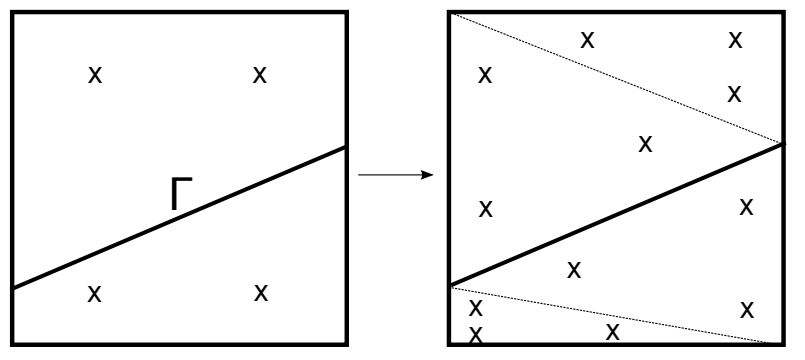

Fig. 2 Geometry subdivision for cut element integration

\subsection{Interface velocity calculation}

The proper evaluation of the interface velocity is crucial in obtaining a precise and robust model. For this particular problem, the interface velocity is determined by the jump in heat flux at the interface, described in (2). The use of a Lagrange multiplier to impose the melting temperature allows the evaluation of the jump in heat flux directly from the Lagrange field $\mathbf{q}$, given by

$$
v_{\Gamma}=\frac{\llbracket \mathbf{q} \rrbracket \cdot \mathbf{n}_{\Gamma}}{\rho L}=\frac{\left(\mathbf{q}_{l}-\mathbf{q}_{s}\right) \cdot \mathbf{n}_{\Gamma}}{\rho L}
$$

where $\mathbf{q}_{s}$ and $\mathbf{q}_{l}$ are the heat fluxes at the interface approaching from the solid and liquid phases, respectively.

The final algorithm can be described as follows. Assuming a given time $t^{n}$, temperature solution $T^{n}$, velocity solution $\mathbf{v}^{n}$, pressure solution $p^{n}$ and level set solution $\phi^{n}$, the strategy to solve for $T^{n+1}, \mathbf{v}^{n+1}$ and $p^{n+1}$ consists in the following steps:

1. Compute the interface velocity $\mathbf{v}_{\Gamma}^{n}$ using (25)

2. Construct $\mathrm{F}$ on the level set domain by solving (24)

3. Solve for $\phi^{n+1}$ using (23)

4. Solve the coupled Stefan-Stokes problem:

4.1. Solve for $T_{i+1}^{n+1}$ using (14) and $\mathbf{v}_{i}^{n+1}$

4.2. Solve for $\mathbf{v}_{i+1}^{n+1}$ and $p_{i+1}^{n+1}$ using (18) and $T_{i+1}^{n+1}$

5. Evaluate (14) and (18). If both residuals are below the tolerance criteria, go to step 6 . If not, $i=i+1$ and go to step 4

6. Set $t^{n+1}=t^{n}$ and go to step 1 .

\subsection{Numerical Integration}

The introduction of discontinuous functions inside elements greatly reduces the precision of standard Gaussian quadrature and may lead to a rank deficient matrix Chessa et al. (2002). An accurate but geometrically complex solution is to subdivide elements involving discontinuities into continuous subelements Moes et al. (1999); Chessa et al. (2002); Gerstenberger and Wall (2010). Each element is subdivided into a number of subelements (lines, triangles or tetrahedrons), as shown in figure 2, to properly fit the contour of the interface (point, line or surface) and element boundaries. The integral over the entire element $I_{e}$ is then the sum of the integration of each subelement $I_{s}$ using standard Hammer quadrature. It is important to note that subelements carry no degrees of freedom or interpolation functions. They are only required as a geometrical tool to construct the element integrals.

In transient problems the location of the quadrature points must change as the interface moves in time, requiring that every cut element be subdivided at each time step. However, the subdivision is applied only to a small number of elements, reducing the overall increase in computational effort required. 
In transient problems, the interpolation functions at time steps $n$ and $n+1$ are based on different positions of the interface and are discontinuous at different places in the element. The integration scheme for the mass matrix (equations (14c) and (18d)) must take both intersections into account when generating the integration subelements to obtain optimal convergence Fries and Zilian (2009). This can be difficult and can significantly increases the number of subelements required to fit the geometry. However, previous authors have successfully used integration schemes considering the current interface position only Chessa et al. (2002); Chessa and Belytschko (2003) and this strategy is used in this work. As suggested in Fries and Zilian (2009), the test functions are evaluated using the current time step's level set values.

\section{RESULTS and DISCUSSIONS}

The Lagrange multiplier formulation used in this work to solve the Stefan problem (1) has been previously validated. For details on the specific simulations used and its performance compared to other algorithms, the interested reader is referred to Martin et al. (2016).

To validate the coupled model two benchmark problems were simulated. The first is the melting of a cylinder inside a channel. The second benchmark problem is the melting of pure tin based on the experimental and numerical data found in Wolff and Viskanta (1988) and the phase change example model found in Comsol Comsol.

In both cases, the same simulation was then run in Comsol, using a moving mesh algorithm to account for the displacement of the interface and was compared with the solution obtained using the purely XFEM approach. The Comsol version was done without any remeshing during the simulation. An appropriate element size was used to maintain a low enough Peclet number to avoid oscillations in the Stefan problem.

These problems were selected for their relatively simple interface geometry and no reinitialization procedure was applied to the level set field during the simulation. For smooth interface shapes and relatively uniform displacements, the absence of a reinitialization step had little impact on the model's accuracy Martin et al. (2016). More complex shapes and interface movements would require a reinitialization step as well as a remeshing step in the Comsol algorithm.

\subsection{Melting cylinder in a channel}

The problem setup is as follows. A channel, $l=0.167 \mathrm{~m}$ in length and $h=0.025 \mathrm{~m}$ in height, contains a solid cylinder of radius $0.005 \mathrm{~m}$. Both phases are initially at the melting temperature $T_{m}=273 \mathrm{~K}$. The cylinder's centre is initially at $\left(\frac{l}{4}, \frac{h}{2}\right)$. At $t=0$, a pressure difference $\Delta p=4 \mathrm{~Pa}$ is applied between the channel's inlet and outlet. The inlet temperature is $274 \mathrm{~K}$. Both top and bottom edges are thermally insulated with a no-slip boundary condition. The pressure difference drives the fluid flow and the buoyancy force was removed from (4a). The material properties used are given in table 1 and a schematic representation of the problem in figure 3. The mesh includes 2904 quadrilateral elements.

The presence of fluid flow around the cylinder increases the heat flux on the top and bottom, where the flow is more rapid. The uphill and downhill sides of the cylinder have a slower fluid flow, leading to a lower heat flux. This results in a more oval shaped interface with time.

The time step used is $\Delta t=1 \mathrm{~s}$ and the tolerance criteria $T_{t o l, v}=$ $10^{8}$ and $T_{t o l, p}=10^{8}$ for the velocity and pressure fields, respectively. The penalty parameter for the Stokes problem is $\beta=10^{8}$. A linear interpolation for both temperature and Lagrange multiplier fields was used.

Figure 4 shows the position of the phase change interface for two different times. Figure 5 shows the evolution of the temperature with time at two points in the domain (see figure 3); one uphill of the cylinder $\left(\mathbf{x}_{1}\right)$ and the other downhill $\left(\mathbf{x}_{2}\right)$. Figure 6 shows the temperature solution in the entire domain at two different times. In all these figures, the Comsol and XFEM models are in excellent agreement. We can observe the change in overall shape of the interface in figure 4, as the fluid flow influences the distribution of heat flux around the cylinder. Furthermore,

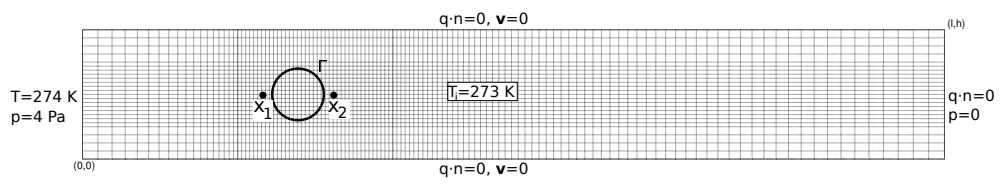

Fig. 3 Problem definition for melting cylinder

Table 1 Material properties for melting cylinder

\begin{tabular}{ll}
\hline Property & Value \\
\hline$\rho\left[\mathrm{kg} / \mathrm{m}^{3}\right]$ & 1000 \\
$T_{m}[\mathrm{~K}]$ & 273 \\
$L[\mathrm{~J} / \mathrm{kg}]$ & $1 \times 10^{8}$ \\
$c_{p}[\mathrm{~J} / \mathrm{kg}]$ & 1000 \\
$k[\mathrm{~W} / \mathrm{m} \cdot \mathrm{K}]$ & 10 \\
$\mu\left[\mathrm{kg} / \mathrm{s} \cdot \mathrm{m}^{2}\right]$ & 0.01 \\
\hline
\end{tabular}

the temperature downhill of the cylinder increases as the cylinder's area decreases and the flow becomes more uniform, as can be seen in figure 6 .

Figure 7 shows the evolution of the velocity with time at points $\mathbf{x}_{1}$ and $\mathbf{x}_{2}$ in the domain (see figure 3 ). Figure 8 shows the velocity solution in the entire domain at two different times. The figures shows that the XFEM solution is in good agreement with the solution obtained with Comsol. We can observe the increase in velocity with time uphill and downhill of the cylinder in figure 8 , as the influence of the cylinder on the fluid flow decreases.

\subsection{Melting of pure tin}

The problem setup for the melting of tin is as follows. A square cavity, $0.10 \mathrm{~m}$ wide and $0.10 \mathrm{~m}$ high, is filled with liquid tin on the left and solid tin on the right. Both phases are initially at the melting temperature $T_{m}=505 \mathrm{~K}$. The initial interface is vertical at $\mathrm{x}=0.02 \mathrm{~m}$. At $t=0$, the temperature of the left wall is increased to $508 \mathrm{~K}$ and the right wall decreased to $503 \mathrm{~K}$, causing the metal to melt. Both top and bottom edges are insulated. The four boundaries are considered walls and a noslip boundary is applied for the Stokes equations. The material properties used are given in table 2 and a schematic representation of the problem in figure 9. The mesh includes 2142 quadrilateral elements ( $42 \times 52$ grid).

The presence of natural convection changes the heat flux within the melt by increasing the influx of heat near the top of the enclosure and reducing it near the bottom, resulting in an angled interface.

The time step used is $\Delta t=3 \mathrm{~s}$ and the tolerance criteria $T_{t o l, v}=$ $10^{1}$ and $T_{t o l, p}=10^{2}$ for the velocity and pressure fields, respectively. The penalty parameter for the Stokes problem is $\beta=10^{8}$. A linear interpolation was used for the temperature and a constant per element $\left(C^{-1}\right.$ continuous) interpolation was used for the Lagrange multiplier.

Figure 10 shows the position of the phase change interface for three different times. Figure 11 shows the evolution of the temperature with time at two points in the domain (see figure 9); one in the liquid phase $\left(\mathbf{x}_{1}\right)$ and the other in the solid phase $\left(\mathbf{x}_{2}\right)$. Figure 12 shows the temperature

Table 2 Material properties melting of pure tin Comsol

\begin{tabular}{ll}
\hline Property & Value \\
\hline$\rho\left[\mathrm{kg} / \mathrm{m}^{3}\right]$ & 7500 \\
$T_{m}[\mathrm{~K}]$ & 505 \\
$L[\mathrm{~J} / \mathrm{kg}]$ & $6 \times 10^{4}$ \\
$c_{p}[\mathrm{~J} / \mathrm{kg}]$ & 200 \\
$k[\mathrm{~W} / \mathrm{m} \cdot \mathrm{K}]$ & 60 \\
$\beta[1 / \mathrm{K}]$ & $2.67 \times 10^{-4}$ \\
$\mu\left[\mathrm{kg} / \mathrm{s} \cdot \mathrm{m}^{2}\right]$ & $6 \times 10^{-3}$ \\
\hline
\end{tabular}




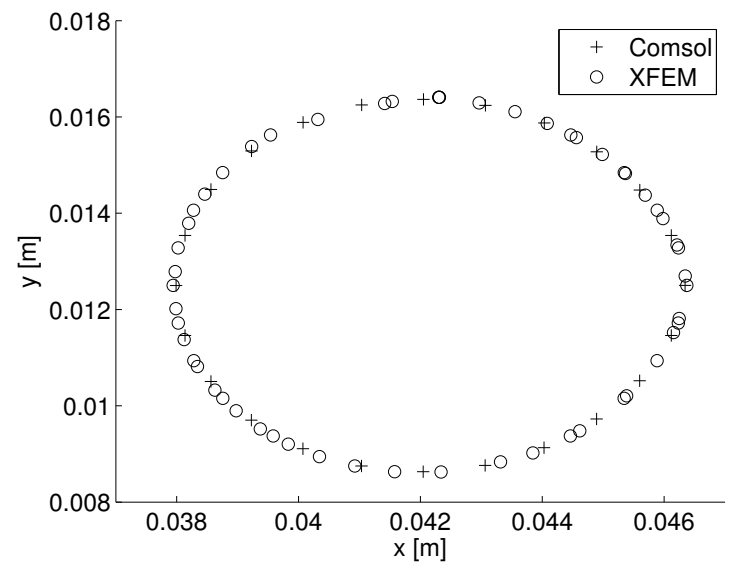

(a) $t=20 \mathrm{~s}$

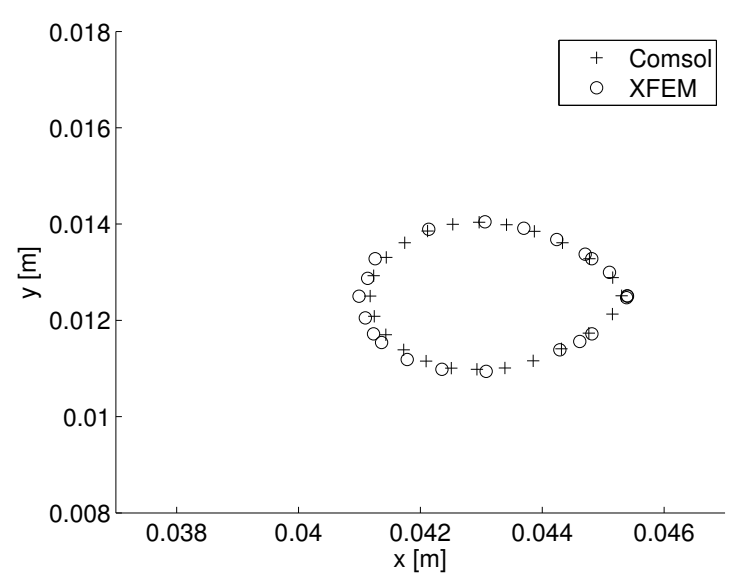

(b) $t=50 \mathrm{~s}$

Fig. 4 Interface position at two times for melting cylinder: (a) $t=20 \mathrm{~s}$, (b) $\mathrm{t}=50 \mathrm{~s}$.

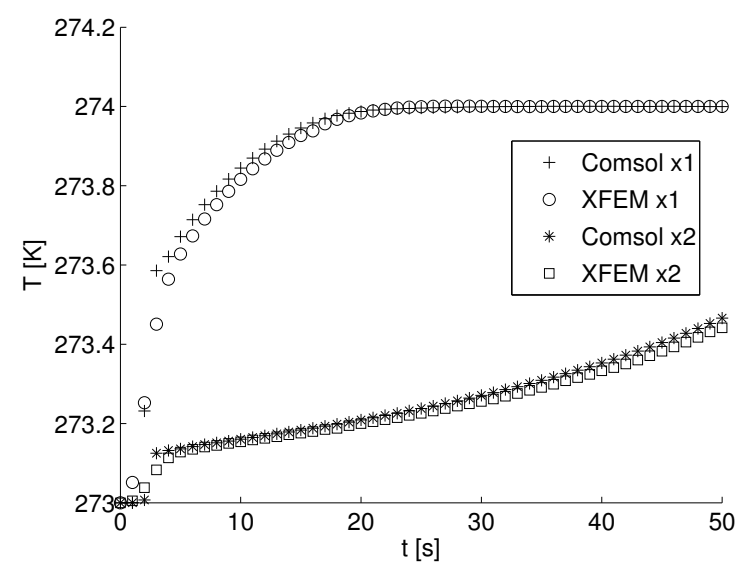

Fig. $5 \mathrm{~T}$ as function of $\mathrm{t}$ at $\mathbf{x}_{1}$ and $\mathbf{x}_{2}$ for melting cylinder (see figure 3)

solution in the entire domain at three different times. In all these figures, the Comsol and XFEM models are in excellent agreement.

There is however in figure 12(a) a small "kink" in the interface position for the XFEM solution. This error is caused by an incorrect evaluation of the interface velocity, due to the use of a constant per element Lagrange multiplier interpolation scheme. As discussed in Martin et al.
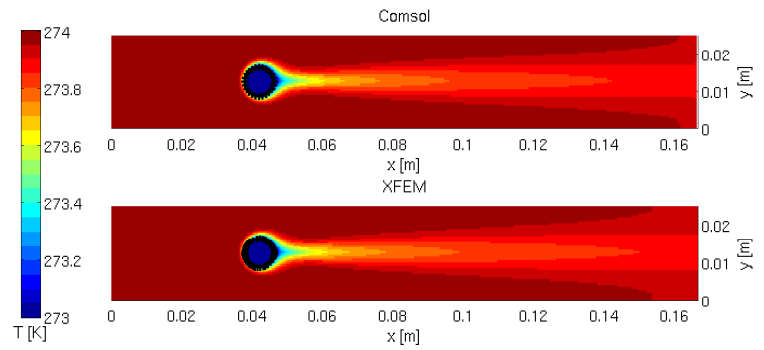

(a) $t=20 \mathrm{~s}$

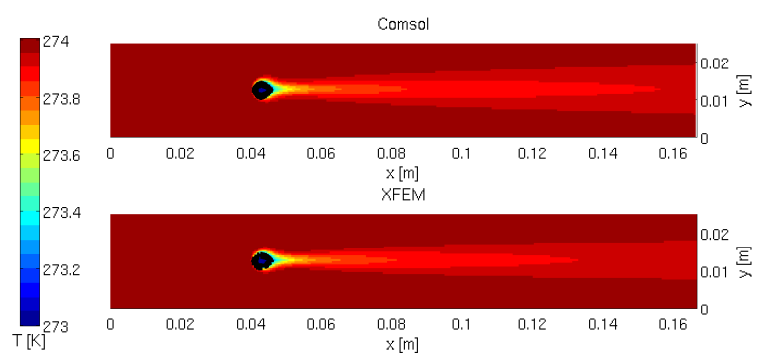

(b) $t=50 \mathrm{~s}$

Fig. 6 Temperature profiles for melting cylinder: (a) $t=20 \mathrm{~s}$, (b) $t=50$ s.

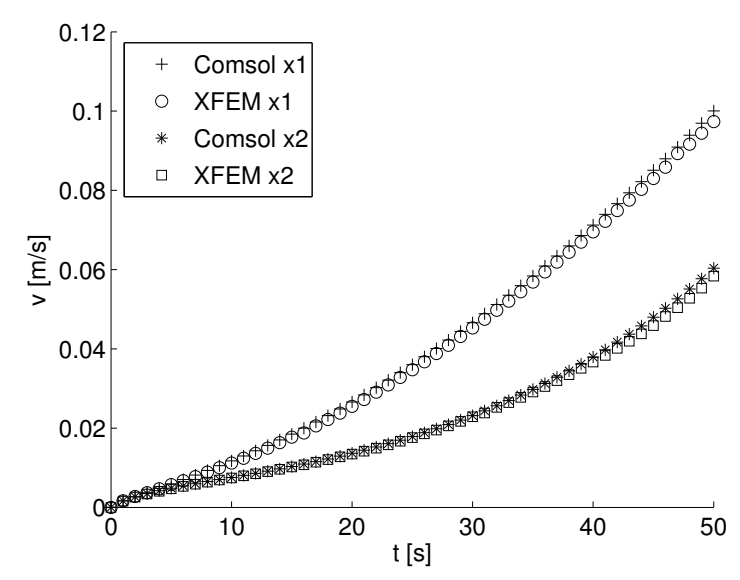

Fig. $7 \mathbf{v}$ as function of t at $\mathbf{x}_{1}$ and $\mathbf{x}_{2}$ for melting cylinder (see figure 3)

(2016), the use of a constant per element interpolation scheme for the Lagrange multiplier is computationally efficient, but not as precise as a linear interpolation. To verify this, the model was run again using a linear interpolation for the multiplier and the kink was no longer present, as shown in figure 13. Furthermore, it is clear from the other figures that the error produced by the use of a constant per element interpolation was quickly corrected over the next few time steps.

Figure 14 shows the evolution of the velocity with time at $\mathbf{x}_{1}$ in the domain (see figure 9). A small difference can be seen between the XFEM and Comsol models at the beginning of the simulation. However, this difference quickly disappears as the simulation advances in time. This initial difference may be caused by the presence of a consistent initialization step by Comsol, which modifies the initial conditions of the problem. Removing this step in the Comsol algorithm lead to a divergent solution. This step is absent in the XFEM model.

Figure 15 shows the velocity solution in the entire domain at three different times. The figure shows that the XFEM solution is in good agreement with the solution obtained with Comsol. The areas with a 

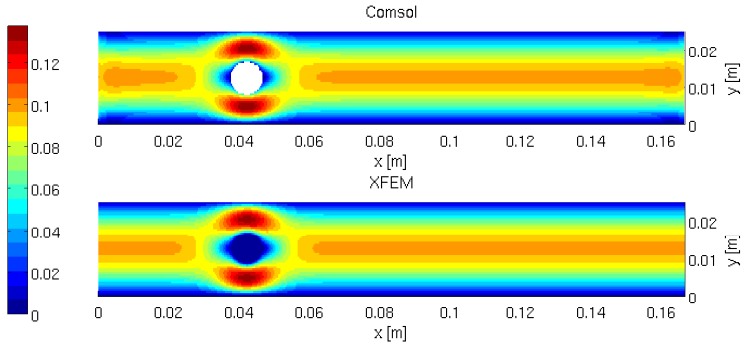

(a) $t=20 \mathrm{~s}$
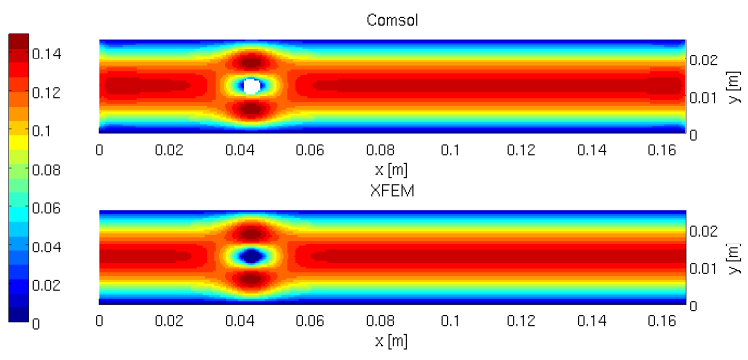

(b) $t=50 \mathrm{~s}$

Fig. 8 Velocity profiles for melting cylinder: (a) $t=20 s$, (b) $t=50 s$.

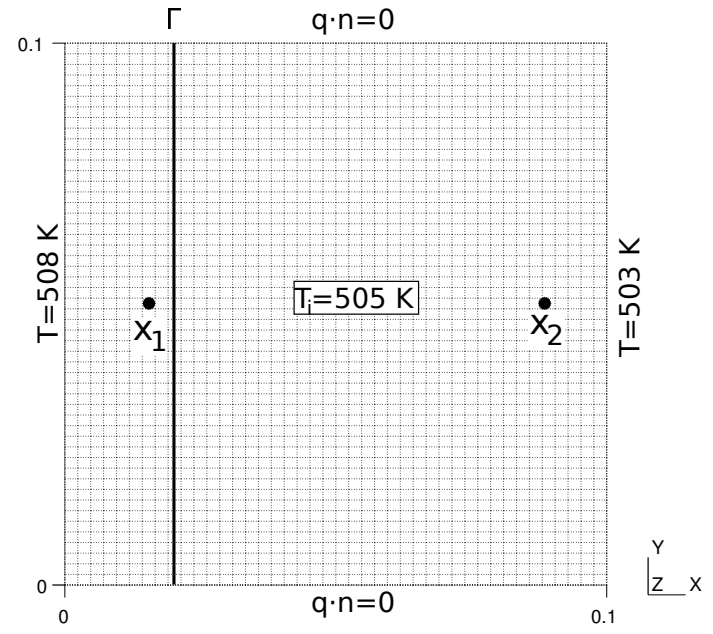

Fig. 9 Problem definition for melting of tin

more horizontal flow have the greatest impact on the temperature distribution (interface position) and are nearly identical. The XFEM formulation however, produces an irregular boundary layer on the solid-liquid interface. This error is caused by the removal of inappropriate degrees of freedom with (21), mainly due to the low tolerance values used. Higher values of $T_{t o l}$ were tried but would lead to a divergent system for certain time steps with critical interface positions. As mentioned previously, the application of a preconditionner Lang et al. (2014) is required to improve the precision of the solution to the Stokes problem.

\section{CONCLUSION}

A coupled Stefan and Stokes formulation using the extended finite element method was developed for the resolution of phase change problems involving convection. The Lagrange multiplier technique developed for the diffusive case was successfully applied to the convective-diffusive case. The temperature and velocity fields obtained using XFEM were compared to the moving mesh algorithm found in Comsol with good results. The XFEM formulation required less degrees of freedom and didn't

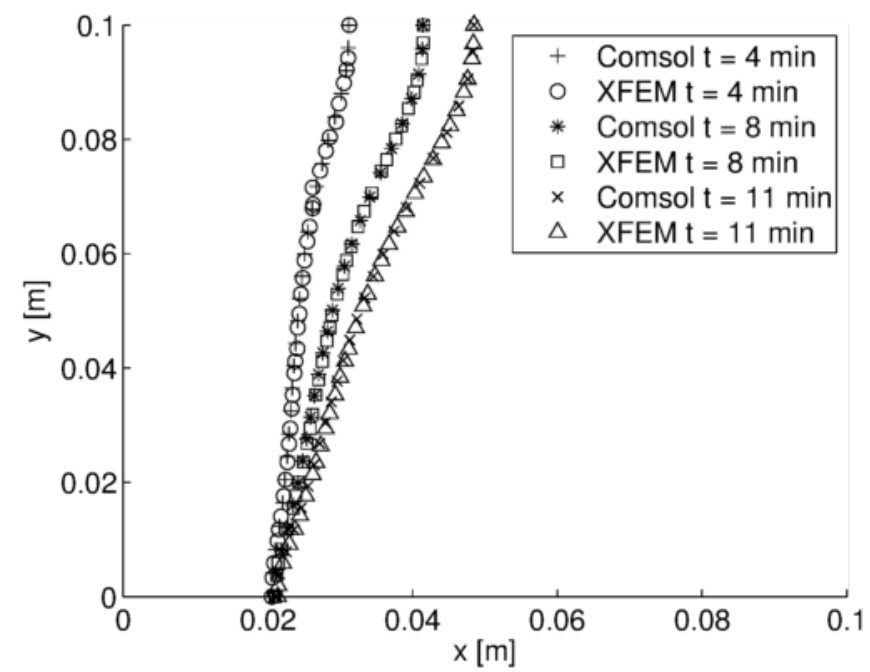

Fig. 10 Interface position for melting tin

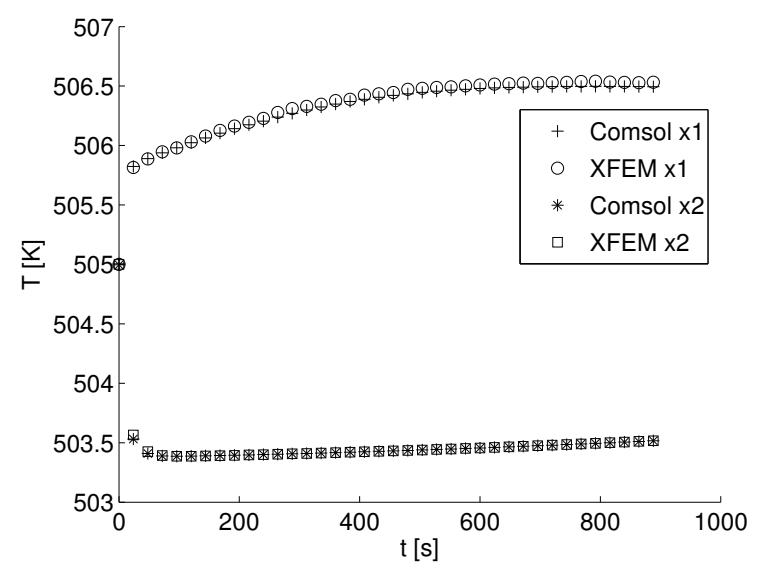

Fig. $11 \mathrm{~T}$ as function of $\mathrm{t}$ at $\mathbf{x}_{1}$ and $\mathbf{x}_{2}$ for melting tin (see figure 9)

cause problems with distorted elements. The tolerance criteria for the removal of degrees of freedom using a void enrichment defined in Lang et al. (2014) was modified for the proposed $\mathrm{Q}_{2}-\mathrm{Q}_{1}$ Stokes formulation. This modified tolerance criteria was shown to produce the same errors in the solution for problematic interface configurations as was observed in Lang et al. (2014). Future work will be done to include the complete Navier-Stokes equations and the application of non-zero velocity boundary conditions on the interface to include density changes between solid and liquid phases.

\section{Acknowledgement}

The authors are grateful for the research support of the Natural Sciences and Engineering Research Council of Canada (File IRSCSA 394855 - 07) and Alcoa. A part of the research presented in this paper was financed by the Fonds de recherche du Québec - Nature et Technologies by the intermediary of the Aluminium Research Centre âĂŞ REGAL. Special thanks to Patrice Goulet for his invaluable assistance with the developpement of the level set algorithm, and advice on the art of code writing. 

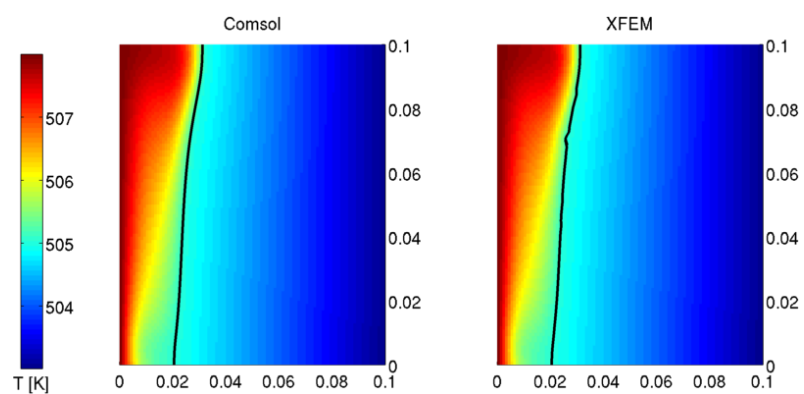

(a) $t=4 \mathrm{~min}$
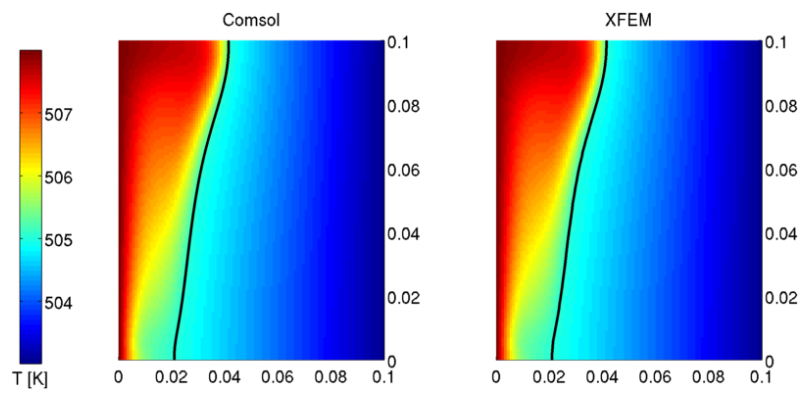

(b) $t=8 \mathrm{~min}$

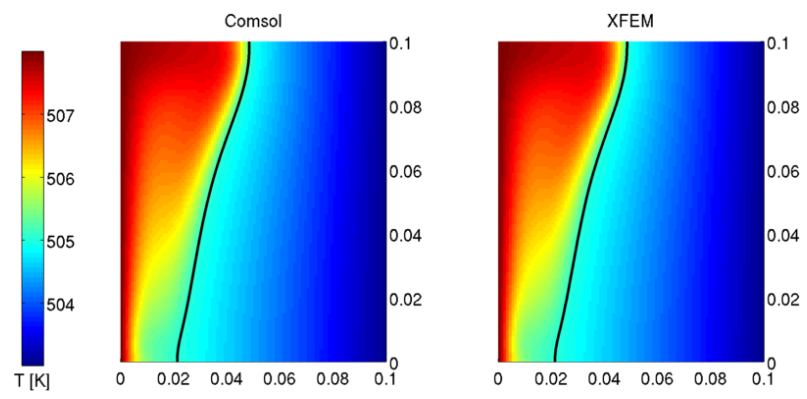

(c) $t=11 \mathrm{~min}$

Fig. 12 Temperature profiles for melting tin: (a) $t=4 \min$, (b) $t=8 \mathrm{~min}$, (c) $\mathrm{t}=11 \mathrm{~min}$.

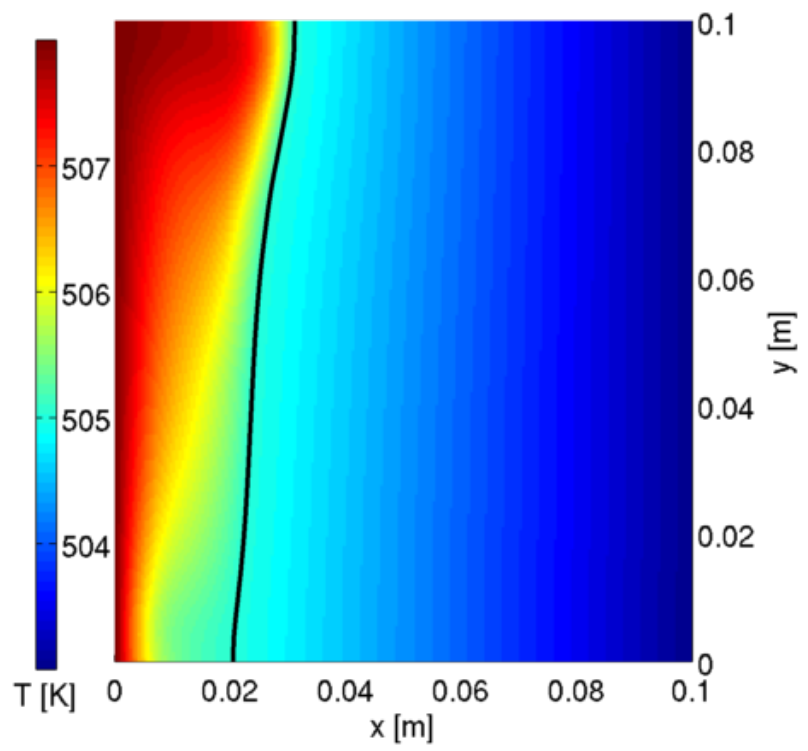

Fig. 13 Temperature profile at $\mathrm{t}=4 \mathrm{~min}$, using linear $\mathbf{q}$

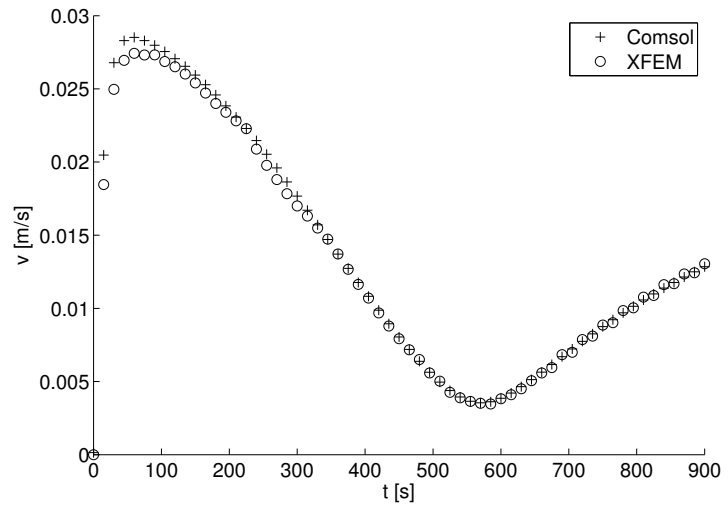

Fig. $14 \mathbf{v}$ as function of $t$ at $\mathbf{x}_{1}$ for melting tin (see figure 9)

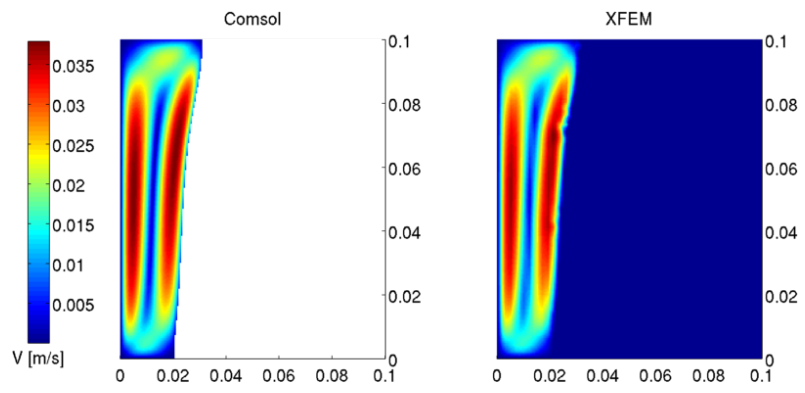

(a) $t=4 \mathrm{~min}$

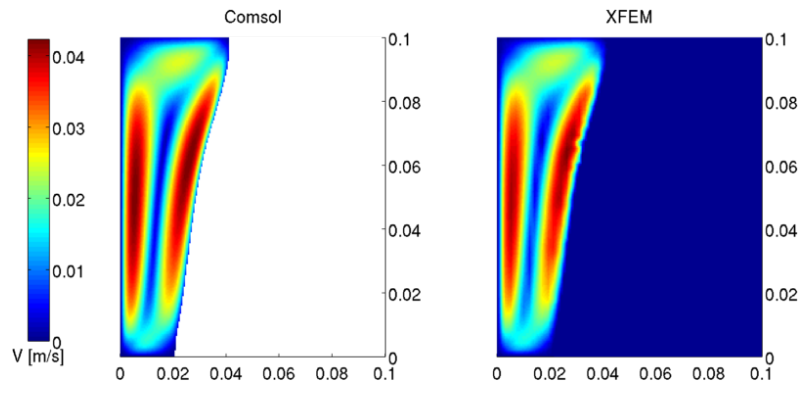

(b) $t=8 \mathrm{~min}$

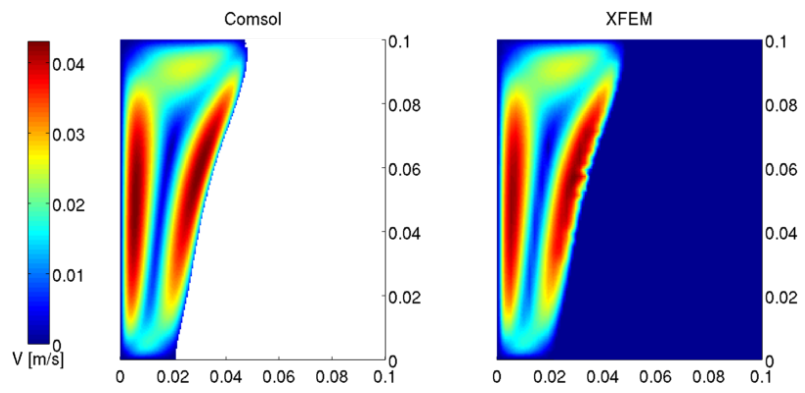

(c) $t=11 \mathrm{~min}$

Fig. 15 Velocity profiles for melting tin: (a) $t=4 \min$, (b) $t=8$ min, (c) $\mathrm{t}=11 \mathrm{~min}$. 


\section{REFERENCES}

Babuska, I., and Melenk, J., 1997, "The Partition of Unity Method," International Journal for Numerical Methods in Engineering, 40(4), 727-758.

http://dx.doi.org/10.1002/(SICI)1097-0207(19970228)40:4<727::AIDNME86>3.3.CO;2-E.

Baiges, J., Codina, R., Henke, F., Shahmiri, S., and Wall, W.A., 2012, "A Symmetric Method for Weakly Imposing Dirichlet Boundary Conditions in Embedded Finite Element Meshes," International Journal for Numerical Methods in Engineering, 90, 636-658.

Beckermann, C., Diepers, H., Steinbach, I., Karma, A., and Tong, X., 1999, "Modeling Melt Convection in Phase-field Simulations of Solidification," Journal of Computational Physics, 154(2), 468-496.

http://dx.doi.org/10.1006/jcph.1999.6323.

Belytschko, T., Moes, N., Usui, S., and Parimi, C., 2001, "Arbitrary Discontinuities in Finite Elements," International Journal for Numerical Methods in Engineering, 50, 993-1013.

http://dx.doi.org/110.1002/1097-0207(20010210)50:4<993::AID-

NME164>3.0.CO;2-M.

Belytschko, T., Gracie, R., and Ventura, G., 2009, "A Review of Extended/generalized Finite Element Methods for Material Modeling," Modelling and Simulation in Materials Science and Engineering, 17.

Bernauer, M., and Herzog, R., 2011, "Implementation of an XFEM Solver for the Classical Two-Phase Stefan Problem," Journal of Scientific Computing, 52, 271-293.

Brent, A., Voller, V., and Reid, K., 1988, "Enthalpy-Porosity Technique for Modeling Convection-Diffusion Phase Change: Application to the Melting of a Pure Metal," Numerical Heat Transfer: An International Journal of Compuation and Methodology, 13:3, 297-318.

Chessa, J., and Belytschko, T., 2003, "An Extended Finite Element Method for Two-Phase Fluids," Journal of Applied Mechanics, 70, 10. http://dx.doi.org/10.1115/1.1526599.

Chessa, J., Smolinski, P., and Belytschko, T., 2002, "The Extended Finite Element Method (XFEM) for Solidification Problems," International Journal for Numerical Methods in Engineering, 53(8), 1959-1977. http://dx.doi.org/10.1002/nme.386.

Comsol, " "http://www.comsol.com/model/tin-melting-front6234," URL http://www.comsol.com/model/ tin-melting-front-6234.

Dolbow, J., Moës, N., and Belytschko, T., 2000, "Discontinuous Enrichment in Finite Elements with a Partition of Unity Method," Finite Elements in Analysis and Design, 36, 235-260.

http://dx.doi.org/10.1016/S0168-874X(00)00035-4.

Fries, T.P., 2008, "A Corrected XFEM Approximation Without Problems in Blending Elements," International Journal for Numerical Methods in Engineering, 75(5), 503-532.

http://dx.doi.org/10.1002/nme.2259.

Fries, T.P., and Zilian, A., 2009, "On Time Integration in the XFEM," International Journal for Numerical Methods in Engineering, 79(1), 6993. http://dx.doi.org/10.1002/nme.2558.

Gerstenberger, A., and Wall, W.A., 2010, “An Embedded Dirichlet Formulation for 3D Continua," International Journal for Numerical Methods in Engineering, 82(5), 537-563.

http://dx.doi.org/10.1002/nme.2755.
Gerstenberger, A., 2010, An XFEM Based Fixed-grid Approach to FluidStructure Interaction, Ph.D. thesis, Technical University of Munich.

Gerstenberger, A., and Wall, W.A., 2008, "An eXtended Finite Element Method/Lagrange Multiplier Based Approach for Fluid-Structure Interaction," Computer Methods in Applied Mechanics and Engineering, 197(19-20), 1699-1714.

http://dx.doi.org/10.1016/j.cma.2007.07.002.

Helenbrook, B.T., 2013, "High-Order Adaptive Arbitrary-LagrangianEulerian (ALE) Calculations of Solidification," Proc. of the ASME FEDSM, vol. 1C.

Hughes, T.J., Franca, L.P., and Hulbert, G.M., 1989, “A New Finite Element Formulation for Computational Fluid Dynamics: VIII. The Galerkin/least-squares method for advective-diffusive equations," Computer Methods in Applied Mechanics and Engineering, 73(2), 173 - 189. http://dx.doi.org/10.1016/0045-7825(89)90111-4.

Ji, H., Chopp, D., and Dolbow, J., 2002, “A Hybrid Extended Finite Element/Level Set Method for Modeling Phase Transformations," International Journal for Numerical Methods in Engineering, 54(8), 1209-1233. http://dx.doi.org/10.1002/nme.468.

Lang, C., Makhija, D., Doostan, A., and Maute, K., 2014, "A Simple and Efficient Preconditioning Scheme for Heaviside Enriched XFEM," Computational Mechanics, 54, 1357-1374.

http://dx.doi.org/10.1007/s00466-014-1063-8, 1312 . 6092.

Martin, D., Chaouki, H., Robert, J.L., Ziegler, D., and Fafard, M., 2016, "A XEFM Lagrange Multiplier Technique for Stefan Problems," Frontiers in Heat and Mass Transfer, 7. http://dx.doi.org/10.5098/hmt.7.31.

Melenk, J., and Babuska, I., 1996, "The Partition of Unity Finite Element Method: Basic Theory and Applications," Computer Methods in Applied Mechanics and Engineering, 139, 289-314.

Merle, R., and Dolbow, J., 2002, "Solving thermal and Phase Change Problems with the Extended Finite Element Method," Computational Mechanics, 28, 339-350.

http://dx.doi.org/10.1007/s00466-002-0298-y.

Moes, N., Dolbow, J., and Belytschko, T., 1999, "A Finite Element Method For Crack Growth Without Remeshing," International Journal for Numerical Methods in Engineering, 46, 131-150.

Nedjar, B., 2002, "An Enthalpy-based Finite Element Method for Nonlinear Heat Problems Involving Phase Change," Computers \& Structures, 80(1), $9-21$.

http://dx.doi.org/10.1016/S0045-7949(01)00165-1.

Osher, S., and Fedkiw, R., 2001, "Level Set Methods: An Overview and Some Recent Results," Journal of Compuational Physics, 169(2), 463502 .

http://dx.doi.org/10.1006/jcph.2000.6636.

Osher, S., and Fedkiw, R., 2003, Level Set Methods and Dynamic Implicit Surfaces, Springer-Verlag.

Osher, S., and Sethian, J.A., 1988, "Fronts Propagating with Curvaturedependent Speed: Algorithms Based on Hamilton-Jacobi Formulations," Journal of Computational Physics, 79(1), 12 - 49. http://dx.doi.org/10.1016/0021-9991(88)90002-2.

Özişik, M.N., 1993, Heat Conduction, John Wiley \& Sons, Inc.

Reddy, J., 2006, An Introduction to the Finite Element Method, 3rd ed., McGraw-Hill. 
Shibanuma, K., and Utsunomiya, T., 2009, "Reformulation of XFEM Based on PUFEM for Solving Problem Caused by Blending Elements," Finite Elements in Analysis and Design, 45(11), 806-816.

http://dx.doi.org/10.1016/j.finel.2009.06.007.

Vynnycky, M., and Kimura, S., 2007, "An Analytical and Numerical Study of Coupled Transient Natural Convection and Solidification in a Rectangular Enclosure," International Journal of Heat and Mass Transfer, 50, 5204-5214.
Wolff, F., and Viskanta, R., 1988, "Soldification of a Pure Metal at a Vertical Wall in the Presence of Liquid Superheat," International Journal of Heat and Mass Transfer, 31, 1735-1744.

Zabaras, N., Ganapathysubramanian, B., and Tan, L., 2006, "Modelling Dendritic Solidification with Melt Convection using the Extended Finite Element Method," Journal of Computational Physics, 218(1), 200-227. http://dx.doi.org/10.1016/j.jcp.2006.02.002. 